\title{
Selective brain cooling achieves peripheral organs protection in hemorrhagic shock resuscitation via preserving the integrity of the brain-gut axis
}

\author{
Chien-Ming Chao ${ }^{1,2}$, Chien-Chin Hsu ${ }^{3}$, Chien-Cheng Huang,3,4, Chung-Han Wang 6 , Mao-Tsun Lin 6 , \\ Ching-Ping Chang ${ }^{\circledR}$, Hung-Jung Lin ${ }^{3,7}, \bowtie$, Chung-Ching Chio ${ }^{8}$ \\ 1. Department of Intensive Care Medicine, Chi Mei Medical Center, Liouying, Tainan, Taiwan \\ 2. Department of Nursing, Min-Hwei College of Health Care Management, Tainan, Taiwan. \\ 3. Department of Emergency Medicine, Chi Mei Medical Center, Tainan, Taiwan \\ 4. Department of Senior Services, Southern Taiwan University of Science and Technology, Tainan, Taiwan. \\ 5. Department of Environmental and Occupational Health, College of Medicine, National Cheng Kung University, Tainan, Taiwan. \\ 6. Department of Medical Research, Chi Mei Medical Center, Tainan, Taiwan \\ 7. Department of Medicine, Taipei Medical University, Taipei, Taiwan. \\ 8. Division of Neurosurgery, Department of Surgery, Chi Mei Medical Center, Tainan, Taiwan.
}

$\square$ Corresponding authors: Ching-Ping Chang, Ph.D., Department of Medical Research, Chi Mei Medical Center. Address: No. 901, Zhonghua Rd, Yongkang District, Tainan City 710, Taiwan. Phone: +886-6-2812811 ext 52657; Fax: +886-6-2832639; E-mail: jessica.cpchang@gmail.com or a50831@mail.chimei.org.tw. Hung-Jung Lin, M.D., Department of Emergency Medicine, Chi Mei Medical Center. Address: No. 901, Zhonghua Rd, Yongkang District, Tainan City 710, Taiwan. Phone: +886-6-2812811 ext 52000; Fax: +886-6-2832639; E-mail: 790001@mail.chimei.org.tw. Chung-Ching Chio, M.D., Division of Neurosurgery, Department of Surgery, Chi Mei Medical Center. Address: No. 901, Zhonghua Rd, Yongkang District, Tainan City 710, Taiwan. Phone: +886-6-2812811 ext 52000; Fax: +886-6-2832639; E-mail: chiocc@ms28.hinet.net.

(c) The author(s). This is an open access article distributed under the terms of the Creative Commons Attribution License (https://creativecommons.org/licenses/by/4.0/). See http://ivyspring.com/terms for full terms and conditions.

Received: 2021.04.02; Accepted: 2021.05.23; Published: 2021.06.04

\begin{abstract}
Background: Although whole-body cooling has been reported to improve the ischemic/reperfusion injury in hemorrhagic shock (HS) resuscitation, it is limited by its adverse reactions following therapeutic hypothermia. $\mathrm{HS}$ affects the experimental and clinical bowel disorders via activation of the brain-gut axis. It is unknown whether selective brain cooling achieves beneficial effects in HS resuscitation via preserving the integrity of the brain-gut axis.

Methods: Male Sprague-Dawley rats were bled to hypovolemic HS and resuscitated with blood transfusion followed by retrograde jugular vein flush (RJVF) with $4{ }^{\circ} \mathrm{C}$ or $36{ }^{\circ} \mathrm{C}$ normal saline. The mean arterial blood pressure, cerebral blood flow, and brain and core temperature were measured. The integrity of intestinal tight junction proteins and permeability, blood pro-inflammatory cytokines, and multiple organs damage score were determined.

Results: Following blood transfusion resuscitation, HS rats displayed gut barrier disruption, increased blood levels of pro-inflammatory cytokines, and peripheral vital organ injuries. Intrajugular-based infusion cooled the brain robustly with a minimal effect on body temperature. This brain cooling significantly reduced the HS resuscitation-induced gut disruption, systemic inflammation, and peripheral vital organ injuries in rats.

Conclusion: Resuscitation with selective brain cooling achieves peripheral vital organs protection in hemorrhagic shock resuscitation via preserving the integrity of the brain-gut axis.
\end{abstract}

Key words: hemorrhagic shock, resuscitation, gut barrier, selective brain cooling

\section{Introduction}

The causes of hemorrhagic shock (HS), a form of hypovolemic shock in which severe blood loss leads to an inadequate oxygen supply, includes trauma, maternal hemorrhage, gastrointestinal hemorrhage, perioperative hemorrhage, and rupture aneurysm [1].
Mild whole-body cooling (WHC) has demonstrated a powerful ability to reduce ischemia-reperfusion injury in HS [2-5]. However, the adverse reactions following therapeutic hypothermia $\left(28-32{ }^{\circ} \mathrm{C}\right)$ limit its use in treating HS resuscitation [6]. In particular, it 
might exacerbate coagulopathy in traumatized patients with HS [7].

Hemorrhagic shock or ischemia/reperfusion affected the experimental and clinical bowel disease via the brain-gut axis activation [8]. Ischemia/ reperfusion after HS severely damaged the gut barrier [9]. This raises the possibility that selective brain might improve outcomes of HS resuscitation by preserving the normality of gut barrier permeability.

Infusion of ice-cold saline through internal jugular vein achieved retrograde cerebral infusion achieved selective brain cooling $\left(32{ }^{\circ} \mathrm{C}\right)$ without affecting body core temperature and provided protective effect in ischemic stroke [10], heat stroke [11], and traumatic brain injury $[12,13]$. However, it is unknown whether the ischemic/reperfusion injury during HS resuscitation can be ameliorated by using selective brain cooling.

The central hypothesis of this study is that intrajugular infusion of cold saline initiated after the onset of blood transfusion resuscitation in a rat HS model will confer peripheral vital organs protection that was achieved by whole-body cooling [2]. Following HS, damage to the intestine and other peripheral organs of rats was assessed histologically and biochemically. In particular, intestinal epithelial permeability, systemic inflammation, and peripheral organ injuries were evaluated.

\section{Materials and Methods}

\section{Animal care}

We obtained adult male Sprague-Dawley rats (weight $350 \pm 10 \mathrm{~g}$ ) from BioLASCO Taiwan Co., Ltd., and housed them at an ambient temperature of $24 \pm 1$ ${ }^{\circ} \mathrm{C}$ with a 12-h light-dark cycle. The Institutional Animal Care and Use Committee (IACUC) of Chi Mei Medical Center approved all animal procedures (IACUC no. 103121505). We used the ARRIVE checklist when writing our report.

\section{A hemorrhagic shock (HS) model}

All animals were intraperitoneally injected with sodium pentobarbital $(40 \mathrm{mg} / \mathrm{kg}$ B. W., SigmaAldrich, St. Louis, MO, USA) before surgery. In our present study, rats were subjected to an HS as detailed previously [2]. In order to induce HS, we withdrew blood (6 mL/100 g B. W.) via the right femoral artery catheter until the mean arterial blood pressure (MABP) dropped to a stable 25 to $30 \mathrm{mmHg}$ for 60 min. After 60 min of HS, we reinfused the shed blood volumes to restore the MABP to its baseline value during a 30-min resuscitation period, followed by selective brain cooling. Sham operation rats underwent similar surgical procedures except for the blood withdrawn and blood transfusion and maintained their MABP within the normal range. Core body temperature (Tco) was continuously monitored in all animals and was maintained at $36 \pm$ $0.2{ }^{\circ} \mathrm{C}$ in rat groups without selective brain cooling using an electric heating pad. Ten minutes after the start of brain cooling (that is, $100 \mathrm{~min}$ after beginning the induction of HS), the catheter was removed, both the external jugular vein and femoral artery were ligated, and the skin was closed. Animals were returned to their home cages at ambient temperature $\left(\mathrm{Ta}, 24^{\circ} \mathrm{C}\right.$ ) and appropriately fed and hydrated. No supplemental cardiopulmonary support was used. Rats that survived to day three of post-HS were considered survivors, and their data were used for analysis. A schematic diagram of the experimental design is shown in Figure 1 A. All the antibodies and commercial kits used in this study are summarized in Supplementary Table S1.

\section{Selective brain cooling by hypothermic retrograde jugular vein flush (RJVF)}

After surgery, a Homeothermic control unit (RightTemp® Temperature Monitor \& Homeothermic Warming Control Module; Kent Scientific, Torrington, CT, USA) was applied to keep the rectal temperature at $37 \pm 0.5^{\circ} \mathrm{C}$. For delivery of cold saline $\left(4{ }^{\circ} \mathrm{C}, 1.7 \mathrm{~mL} / 100 \mathrm{~g} \mathrm{~B} . \mathrm{W}\right)$ or control vehicle $\left(36{ }^{\circ} \mathrm{C}\right.$, $1.7 \mathrm{~mL} / 100 \mathrm{~g}$ B.W.), cannulas (PE50) were inserted into the right external jugular (EJ) vein (with cranial direction). Within the 10-minutes delivery of cold saline, brain temperature was decreased in the rats of the $4{ }^{\circ} \mathrm{C}$ RJVF, but their body core temperature remained unchanged [11]. After 10-minutes of delivery of cold saline, the brain temperature was maintained at $32-33^{\circ} \mathrm{C}$ for at least 50 minutes. Then, the brain temperatures returned gradually to their original levels (as shown in Figure 1).

\section{Experimental groups}

The rats $(n=78)$ were randomized into the following three groups: (i) sham control rats without HS and resuscitation (R) (non-HS+non-R+RJVF $36^{\circ} \mathrm{C}$; $\mathrm{n}=26$ ); (ii) rats with $\mathrm{HS}, \mathrm{R}$, and RJVF $36{ }^{\circ} \mathrm{C}$ (HS+R+RJVF $36{ }^{\circ} \mathrm{C}$; $\mathrm{n}=26$ ); (iii) rats with HS, $\mathrm{R}$, and RJVF $4{ }^{\circ} \mathrm{C}\left(\mathrm{HS}+\mathrm{R}+\mathrm{RJVF} 44^{\circ} \mathrm{C} ; \mathrm{n}=26\right)$. The saline $\left(36^{\circ} \mathrm{C}\right.$ or $4{ }^{\circ} \mathrm{C}$ ) was administered via cannulas in the external jugular vein using a syringe pump (KD Scientific Inc., MA, USA). On day 3 post-surgery, animals were subjected to the last behavioral tests. Then they were euthanized with an overdose of Zoletil $(100 \mathrm{mg} / \mathrm{kg}$ body weight), and 10\% Xylazine and their organ were removed for histological and biochemical examinations. The overall survival up to 3 days were approximately 10, 8, and 9 out of 10 for non-HS+non-R+RJVF $36{ }^{\circ} \mathrm{C}$, HS+R+RJVF $36^{\circ} \mathrm{C}$, and 
$\mathrm{HS}+\mathrm{R}+\mathrm{RJVF} 4^{\circ} \mathrm{C}$, respectively. Experimenters were blinded as to group allocation.

\section{Measurement of striatal cerebral blood flow (CBF) in rats under general anesthesia}

Upon placing in a stereotaxic apparatus (Model 902, David Kopf Instruments, CA, USA), we inserted an optical probe $\left(300 \mu \mathrm{m}\right.$ tip diameter; OxyLite ${ }^{\mathrm{TM}}$ and OxyFlo $^{\mathrm{TM}}$ system; Oxford Optronix Ltd., UK) into the right striatum using coordinates: $\mathrm{AP},+0.7 \mathrm{~mm}$ distance after the fontanelle; $\mathrm{R},+0.3 \mathrm{~mm}$ movement to the right side; DV, $6 \mathrm{~mm}$ depth value from the top of the skull for monitoring both striatal temperature and striatal CBF with a laser Doppler flowmetry (Oxford Optronix Ltd., UK)[14].

\section{Pathohistological and biochemical studies of other vital organs}

We evaluated the degrees of brain, lung, liver, kidney, and heart tissue damage according to the descriptions detailed previously [2]. We determined the plasma concentrations of blood urea nitrogen (BUN), creatinine, aspartate aminotransferase (AST), alanine aminotransferase (ALT), alkaline phosphatase (ALP), and lactate dehydrogenase (LDH) to assess the renal and hepatic functions and the serum concentrations of tumor necrosis factor- $\alpha$ (TNF- $\alpha$ ), interleukin-1 $\beta$ (IL-1 $\beta$ ), interleukin-6 (IL-6), and interleukin-18 (IL-18) to assess the inflammatory status.

\section{Intestinal damage scores and intestinal permeability determination}

The duodenum, jejunum, and terminal ileum were fixed with $10 \%$ buffered formaldehyde and embedded in paraffin, sliced, stained with hematoxylin and eosin (H\&E), and then evaluated the degree of HS-induced damage [15].

Intestinal permeability was determined by measuring the intestinal clearance of fluoresceinisothiocyanate dextran (FD-4; Sigma-Aldrich) as reported in a previous study [16]. Briefly, $5 \mathrm{~cm}$ segments of duodenum, jejunum, and terminal were collected, and the mucosa was gently everted from each. The intestinal segment was ligated at one end, and from the other end, a gut sac was prepared by injection of $1.0 \mathrm{~mL}$ of Krebs-Henseleit bicarbonate buffer. The filled sac was then incubated in a solution containing $0.5 \mathrm{mg} / \mathrm{mL}$ FD4 (average molecular weight: 4000 ) at $37^{\circ} \mathrm{C}$. The bathing solution was also aerated by gently bubbling with a gas mixture containing $5 \% \mathrm{CO}_{2}$ and $95 \% \mathrm{O}_{2}$. The mucosal-toserosal clearance (C) of FD4 was determined in $\mu \mathrm{L}-\mathrm{min}^{-1} \cdot \mathrm{cm}^{-2}$.
Determination of ZO-1 and claudin-1 immunofluorescence staining at tight junctions of the intestinal epithelium

Intestinal tissues were fixed in $10 \%$ formalin overnight and embedded in paraffin. Ten-micrometer intestinal sections mounted on coated glass slides were deparaffinized, rehydrated, and washed with PBS. Antigen retrieval was performed in $0.01 \mathrm{M}$ citrate buffer (pH 6.0) for $30 \mathrm{~min}$ in a microwave oven. Slides were incubated with peroxidase blocking reagent (Dako, Carpinteria, CA, USA) for $30 \mathrm{~min}$ to block endogenous peroxidase activity and then stained with antibodies targeting claudin-1 (1:200, \#2H10D10, Invitrogen ${ }^{\mathrm{TM}}$, Thermo Fisher Scientific Inc., Waltham, MA, USA) and ZO-1 (1:200, \#ab96587, Abcam, Cambridge, MA, USA) at $4{ }^{\circ} \mathrm{C}$ overnight to detect epithelial tight junction proteins as detailed previously [17]. Slides were then thoroughly washed with PBS and incubated with Alexa Fluor 488 goat anti-mouse IgG antibody (1:400, \# A11008, Invitrogen ${ }^{\mathrm{TM}}$, Thermo Fisher Scientific Inc., Waltham, MA, USA) and Alexa Fluor 568 goat anti-rabbit IgG antibody (1:400, \#A11004, Invitrogen ${ }^{\mathrm{TM}}$, Thermo Fisher Scientific Inc., Waltham, MA, USA) for $1 \mathrm{~h}$ at room temperature. The samples were next counterstained with 4'6-diamidino-2-phenylindole (1:5000, \#D1306, Invitrogen ${ }^{\mathrm{TM}}$, Thermo Fisher Scientific Inc) for $10 \mathrm{~min}$. After a final wash with PBS, slides were mounted in glycerol gelatine mounting medium (\#GG1-15 ML, Sigma-Aldrich, St. Louis, MO, USA) and viewed using an upright fluorescence microscope (Carl Zeiss Microscopy GmbH, Jena, Germany) at excitation/emission wavelengths of 578/603 nm (rhodamine, red) and 490/525 nm (FITC, green). A digital camera linked to a computer running Axioscope version 4 (Carl Zeiss) was used to capture images. A pathologist counted the number of ZO-1/DAPI and claudin-1/DAPI double-labeled cells in 20 frames from five sections (x 400 magnification). All cell counting was performed in a blinded manner to avoid bias.

\section{Western Blot Analysis of Intestinal Tight Junction Proteins Expression}

The concentration of the total protein lysates by homogenizing duodenum tissues ( $\mathrm{n}=6$ in each group) in RIPA (radioimmunoprecipitation assay) lysis buffer with protease and phosphatase inhibitor (Thermo Fischer Scientific Inc., Waltham, MA, USA) were determined with a protein assay kit (Bio-Rad, Hercules, CA, USA). Twenty micrograms of each protein sample were analyzed by $6 \%$ or $12 \%$ sodium dodecyl sulfate-polyacrylamide (SDS-PAGE) gels and then transferred to polyvinylidene fluoride (PCDF) membranes. Subsequently, PVDF membranes were 
incubated with a specific primary antibody to detect rat claudin-1 or ZO-1 protein (\#ab15098, \#ab96587, Abcam, Cambridge, MA, USA) overnight at $4{ }^{\circ} \mathrm{C}$. $\beta$-actin (\#sc47778, Santa Cruz, CA, USA) was probed as a protein loading control. For secondary antibody, anti-rabbit IgG or anti-mouse IgG conjugated to horseradish peroxidase (HRP) (Cell Signaling Technology, Danvers, MA, USA). Band densities were determined by scanning densitometry (GS-800, BIO-RAD, Hercules, CA, USA). Finally, the signals were detected using enzyme-linked enhanced chemiluminescence (ECL) reagent (Thermo Fischer Scientific Inc., \#34080, Waltham, MA, USA). The density of Western blot bands was quantified using an image analysis system (Image Pro-Plus; Media Cybernetics, USA). Claudin-1 and ZO-1 protein levels were determined after normalizing with $\beta$-actin.

\section{Statistics}

Data are presented as the mean \pm S.D. Repeated measures analysis of variance was used to compare serial biochemical data and vital signs. For physiological, behavioral, and biochemical parameters, the data were analyzed by two-way repeated factor analysis of variance. When the analysis of variance showed significant variance, the Tukey-Kramer post hoc test was used. Parameters such as histological scores and the immune fluorescence staining data with non-normal distribution were analyzed by the Kruskal-Wallis test with Dunn's post-hoc test. We used GraphPad Prism (version 7.01 for Windows; GraphPad Software, San Diego, CA, USA) to analyze the data and set the statistically significant level at $\mathrm{P}<0.05$.

\section{Results}

\section{RJVF $4{ }^{\circ} \mathrm{C}$ but not RJVF $36^{\circ} \mathrm{C}$ causes selective brain cooling in HS rats}

As shown in Figure 1, before HS or sham operation, all three groups of rats had normal environment and physiological values, including colon temperature (Tco, Figure 1B), brain temperature in the striatum ( $\mathrm{Tb}$, Figure $1 \mathrm{C})$, mean arterial blood pressure (MABP, Figure 1D), heart rate (HR, Figure 1E), and striatal blood flow (SBF, Figure 1F). However, $60 \mathrm{~min}$ after HS intervention, compared to the non-HS+non-R+RJVF $36{ }^{\circ} \mathrm{C}$ group rats, the two groups of HS rats displayed hypotension, bradycardia, and severe cerebral ischemia (Figure 1). In particular, compared to the non-HS+non-R+RJVF $36{ }^{\circ} \mathrm{C}$ group rats, the $\mathrm{HS}+\mathrm{R}+\mathrm{RJVF} 36^{\circ} \mathrm{C}$ group rats had a significantly lower percentage of SBF (Figure 1F). Immediately after $30 \mathrm{~min}$ of resuscitation by blood transfusion, all physiological parameters were restored to pre-HS levels. In RJVF-treated group rats, immediately after resuscitation, an RJVF with saline at either $36{ }^{\circ} \mathrm{C}$ or $4{ }^{\circ} \mathrm{C}$ was performed for $10 \mathrm{~min}$. Ten minutes of RJVF $4{ }^{\circ} \mathrm{C}$, but not RJVF $36^{\circ} \mathrm{C}$, caused selective brain cooling ( $\mathrm{Tb}$ decreased to $\sim 32{ }^{\circ} \mathrm{C}-33^{\circ} \mathrm{C}$ ) for approximately $50 \mathrm{~min}$ (Figure 1C).

\section{Selective brain cooling attenuates HS-induced intestinal damage and intestinal epithelial hyperpermeability}

Compared to the non-HS+non-R+RJVF $36{ }^{\circ} \mathrm{C}$ group rats, the $\mathrm{HS}+\mathrm{R}+\mathrm{RJVF} 36^{\circ} \mathrm{C}$ group rats had significantly higher intestinal damage scores (Figure 2A-B). In addition, intestinal epithelial permeability evaluated by both the intestinal clearance of FD4 (Figure 2C), the fluorescence intensity of specific intestinal epithelium tight junction proteins (e.g., ZO-1 and claudin-1) (Figure 3A), and protein expression levels (Figure 3B and 3C) in the HS+R+RJVF $36{ }^{\circ} \mathrm{C}$ group rats were also significantly lower than those in non-HS+non-R+RJVF $36^{\circ} \mathrm{C}$ group rats. The increased intestinal damage scores, the intestinal epithelial hyperpermeability, and breakdown of tight junction proteins shown in the $\mathrm{HS}+\mathrm{R}+\mathrm{RJVF} 36^{\circ} \mathrm{C}$ group of rats were all significantly attenuated by selective brain cooling (HS+R+RJVF 4 ${ }^{\circ} \mathrm{C}$ group)(Figure 2 and 3 ).

\section{Selective brain cooling attenuates multiple vital organs damage in HS rats}

Compared to the non-HS+non-R+RJVF $36{ }^{\circ} \mathrm{C}$ group rats, both the $\mathrm{HS}+\mathrm{R}+\mathrm{RJVF} 36^{\circ} \mathrm{C}$ group rats had significantly higher values of lung, liver, kidney, and heart damage scores (Figure 4) and multiple organs damage indicators (e.g., creatinine, BUN, AST, ALT, ALP, and LDH) (Table 1). These increased plasma levels of multiple organ damage markers were all significantly reduced by RJVF $4{ }^{\circ} \mathrm{C}$ saline but not affected by RJVF $36^{\circ} \mathrm{C}$ saline.

Table 1. Indicators of renal, hepatic, and inflammatory function three days after HS or sham intervention.

\begin{tabular}{|c|c|c|c|}
\hline & & \\
\hline \multirow[t]{2}{*}{ Indicators } & non-HS+non-R+RJVF & $\mathrm{HS}+\mathrm{R}+\mathrm{RJVF}$ & \multirow{2}{*}{$\begin{array}{l}\text { HS+R+RJVF } \\
4{ }^{\circ} \mathrm{C}\end{array}$} \\
\hline & $36^{\circ} \mathrm{C}$ & $36^{\circ} \mathrm{C}$ & \\
\hline creatinine $(\mathrm{mg} / \mathrm{dL})$ & $0.38 \pm 0.03$ & $0.79 \pm 0.06^{*}$ & $0.42 \pm 0.04+$ \\
\hline BUN (mg/dL) & $15 \pm 1$ & $31 \pm 5^{*}$ & $15 \pm 3+$ \\
\hline AST (U/L) & $96 \pm 6$ & $319 \pm 14^{*}$ & $82 \pm 6+$ \\
\hline $\operatorname{ALT}(\mathrm{U} / \mathrm{L})$ & $57 \pm 6$ & $187 \pm 19^{*}$ & $71 \pm 3+$ \\
\hline $\operatorname{ALP}(\mathrm{U} / \mathrm{L})$ & $166 \pm 53$ & $686 \pm 89^{*}$ & $219 \pm 17+$ \\
\hline LDH (IU/L) & $177.66 \pm 22.94$ & $525 \pm 99^{*}$ & $288 \pm 22+$ \\
\hline $\mathrm{TNF}-\alpha(\mathrm{pg} / \mathrm{ml})$ & $10 \pm 1$ & $32 \pm 3^{*}$ & $10 \pm 1+$ \\
\hline $\mathrm{IL}-1 \beta(\mathrm{pg} / \mathrm{ml})$ & $16 \pm 2$ & $29 \pm 2^{*}$ & $11 \pm 1+$ \\
\hline IL-6 (pg/ml) & $4 \pm 2$ & $22 \pm 6^{*}$ & $4 \pm 2+$ \\
\hline IL-18 (pg/ml) & $20 \pm 6$ & $59 \pm 6^{*}$ & $36 \pm 7+$ \\
\hline \multicolumn{4}{|c|}{$\begin{array}{l}\text { Values are mean } \pm \text { S.D. ( } n=10 \text { group). }{ }^{*} P<0.05 \text { vs. the non-HS+non-R+RJVF } 36{ }^{\circ} \mathrm{C} \text { group; }{ }^{+} P<0.05 \text { vs. } \\
\text { the HS+R+RJVF } 36^{\circ} \mathrm{C} \text { group. HS, hemorrhagic shock; R, resuscitation; RJVF, retrograde jugular } \\
\text { vein flush; BUN, blood urea nitrogen; AST, aspartate aminotransferase; ALT, alanine } \\
\text { aminotransferase; ALP, alkaline phosphatase; LDH, lactate dehydrogenase; TNF- } \alpha \text {, tumor necrosis } \\
\text { factor-alpha; IL-1 } 1 \beta \text {, interleukin-1beta; IL- } 6 \text {, interleukin-6; IL-18, interleukin- } 18 \text {. }\end{array}$} \\
\hline
\end{tabular}


Selective brain cooling attenuates the overexpression of pro-inflammatory cytokines in $\mathrm{HS}$ rats

Compared to the non-HS+non-R+RJVF $36^{\circ} \mathrm{C}$ group rats, the $\mathrm{HS}+\mathrm{R}+\mathrm{RJVF} 36^{\circ} \mathrm{C}$ group rats had significantly higher blood levels of pro-inflammatory cytokines (e.g., IL-1 $\beta$, TNF- $\alpha$ and IL-18) (Table 1). The overproduction of pro-inflammatory cytokines was significantly reduced by RJVF $4{ }^{\circ} \mathrm{C}$ saline.

(A)

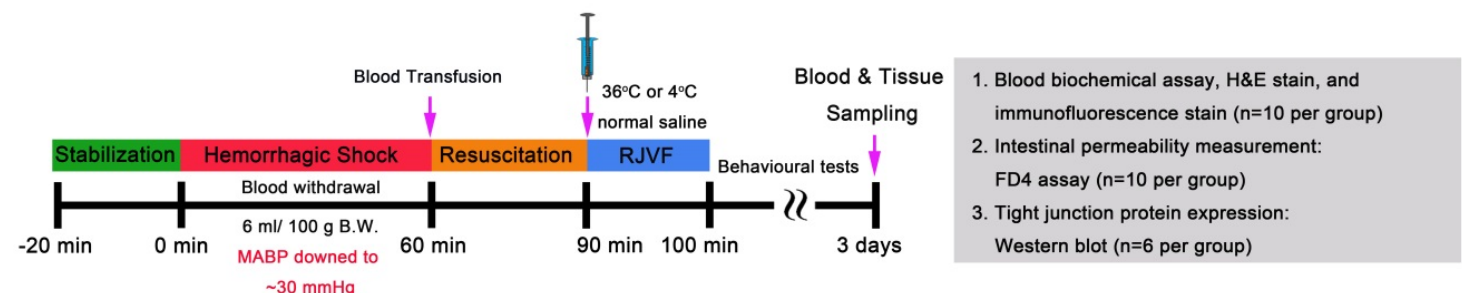

(B)

(C)

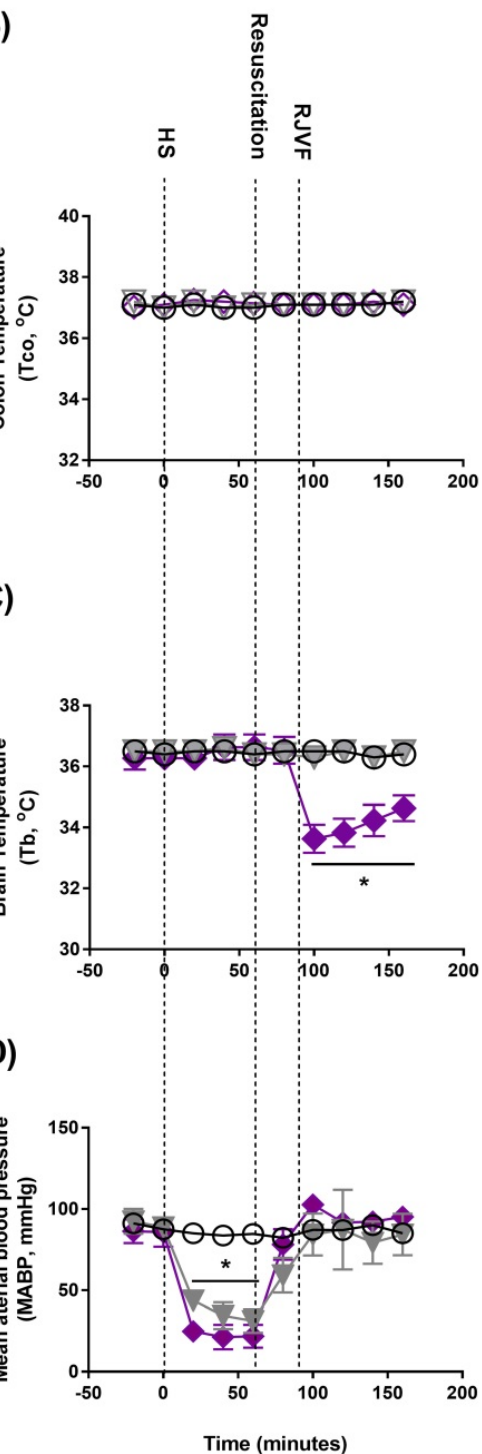

(E)

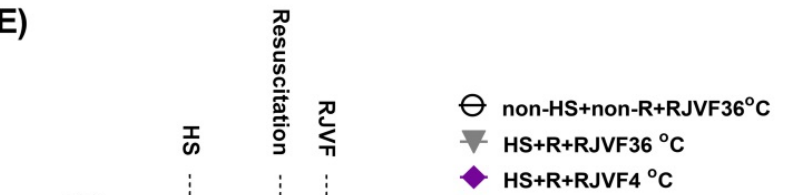

(F)
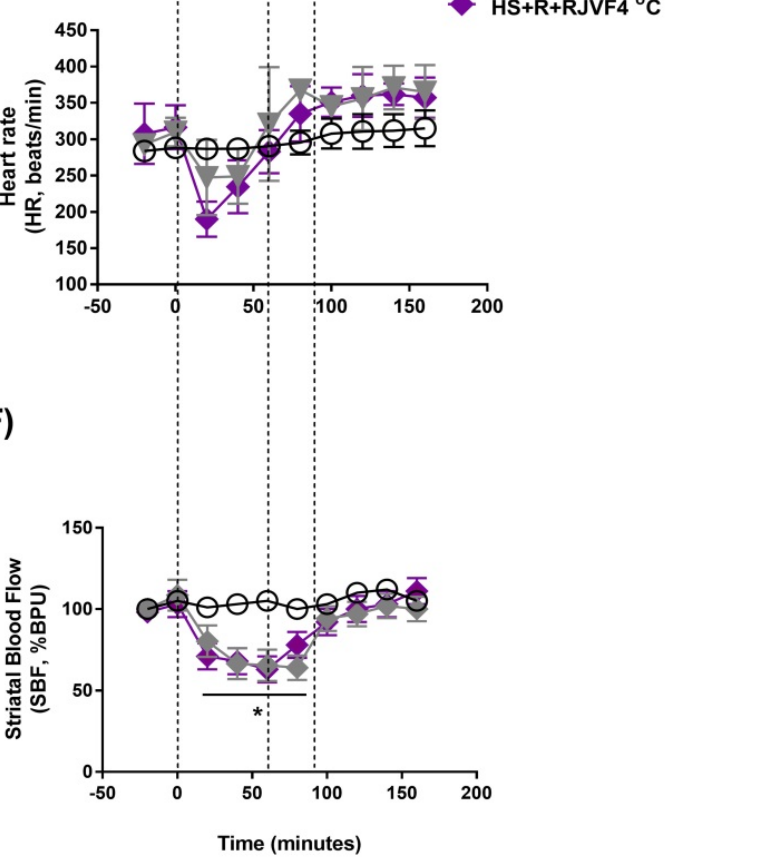

Figure 1. RJVF $4{ }^{\circ} \mathrm{C}$ but not RJVF $36^{\circ} \mathrm{C}$ causes selective brain cooling in HS rats. (A) Illustrations are showing the overall experimental design for the hemorrhagic shock (HS) rat model. (B) to (F) illustrates (B) colon temperature (Tco), (C) brain temperature (Tb), (D) mean arterial blood pressure (MABP), (E) heart rate (HR), (F) striatal blood flow (SBF) for different groups of rats $(n=10)$. ${ }^{*} p<0.01$, compared to the non-HS+non-R+RJVF $36^{\circ} \mathrm{C}$ group. The rats ( $\mathrm{n}=78$ ) were divided into 3 groups ( $\mathrm{n}=26$ for each group: 10 rats for blood biochemical assay, HE stain, and immunofluorescence stain; 10 rats for intestinal permeability measurement by FD4 assay; 6 rats for intestinal tight junction protein assay by Western blotting;). The overall survival up to 3 days were approximately 10,8 , and 9 out of 10 for non-HS+non-R+RJVF $36^{\circ} \mathrm{C}$, HS+R+RJVF $36^{\circ} \mathrm{C}$, and $\mathrm{HS}+\mathrm{R}+\mathrm{RJVF} 4^{\circ} \mathrm{C}$, respectively. 
(A)

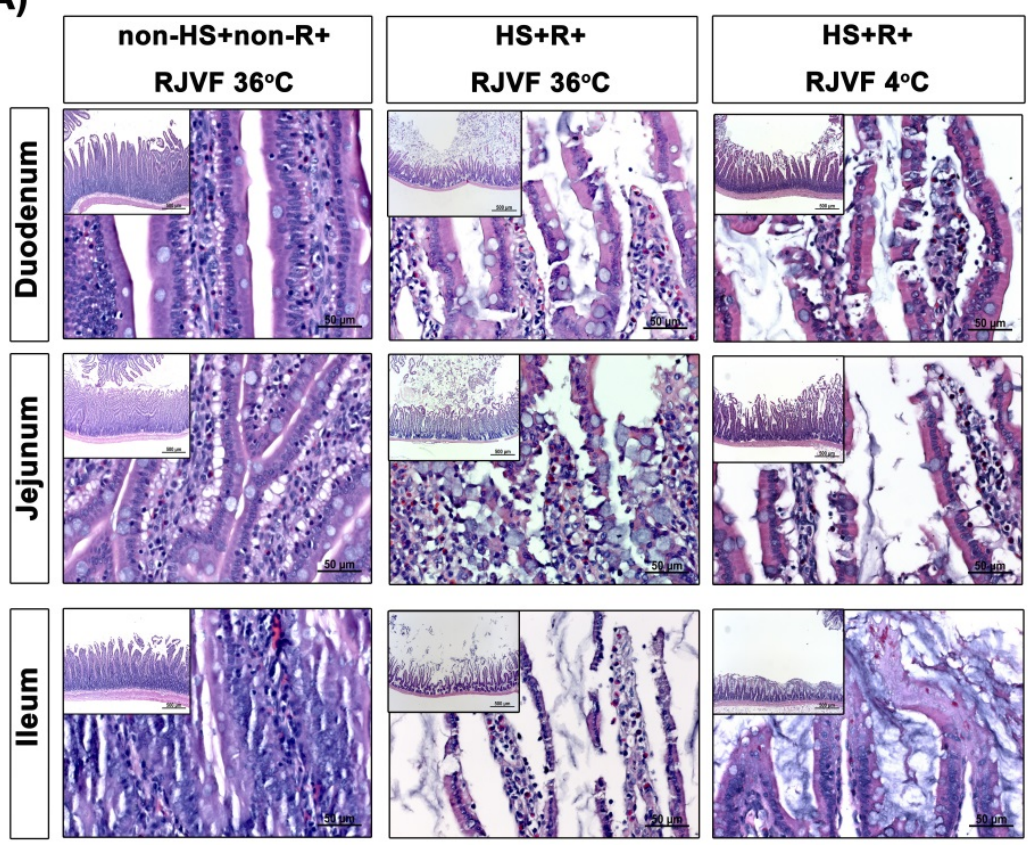

(B)

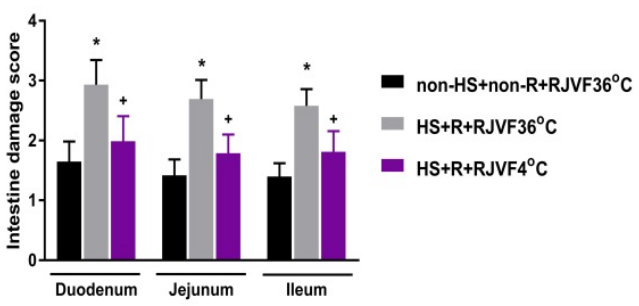

(C)

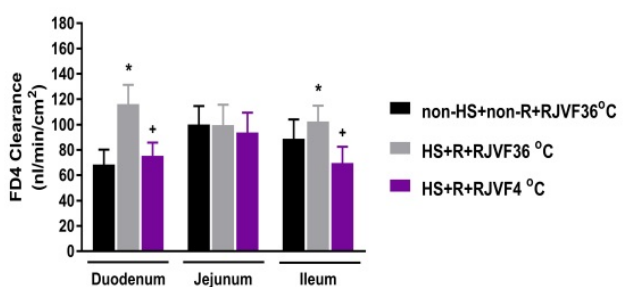

Figure 2. Selective brain cooling attenuates HS-induced intestinal damage and intestinal epithelial hyperpermeability. Representative images of the changes to intestinal tissue induced by HS and RJVF as visualized with H\&E light microscopy (A) and intestinal damage scores (B) in each experimental group. (C) The intestinal clearance of FD4. Scale bar $=50 \mu \mathrm{m}$. The bar graph represents the mean $\pm S . D$. of 10 rats for each group. ${ }^{*} p<0.05$, the HS+R+RJVF $36{ }^{\circ} \mathrm{C}$ group vs. the non-HS+non-R+RJVF $36^{\circ} \mathrm{C}$ group; ${ }^{+} p<0.05$, the $H S+R+R J V F 4{ }^{\circ} \mathrm{C}$ vs. the HS+R+RJVF $36^{\circ} \mathrm{C}$ group.

(A) ZO-1 + Claudin-1 + DAPI
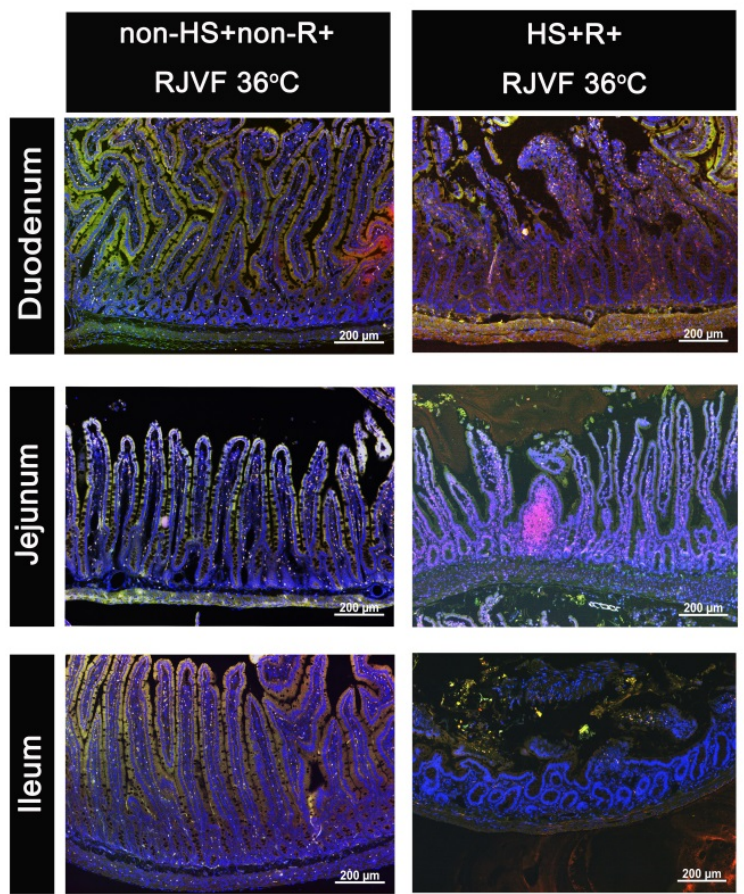

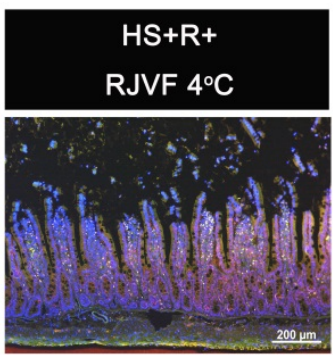

(B)

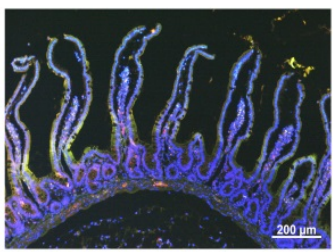

(C)

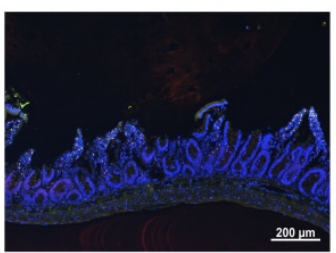

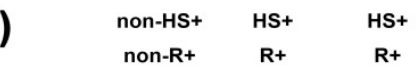
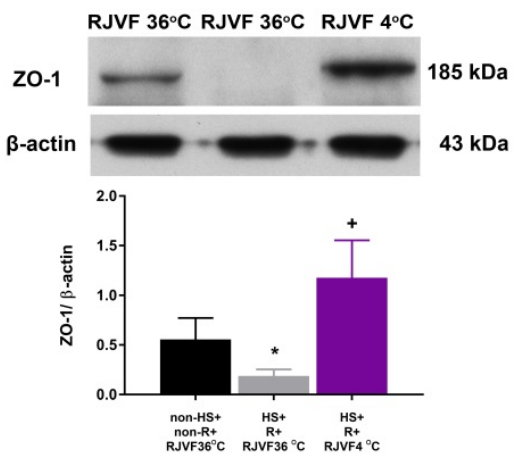

non-HS+ HS+ HS+ non-R+ R+ R+ RJVF $36^{\circ} \mathrm{C}$ RJVF $36^{\circ} \mathrm{C}$ RJVF $4^{\circ} \mathrm{C}$

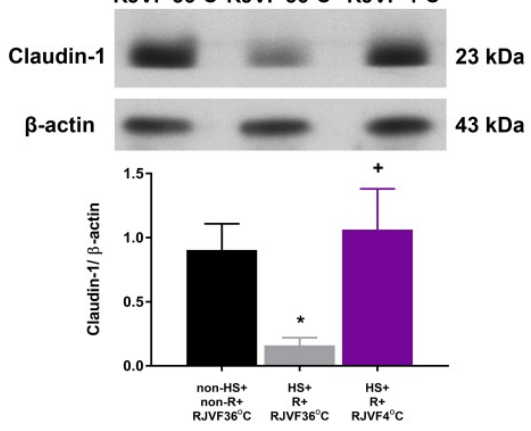

Figure 3. Tight junction protein expression after hemorrhagic resuscitation. Representative images of paraffin sections of the intestine labeled for nuclei (blue), (A) ZO-1 (green), claudin-1 (red), and merged (yellow). ZO-1 and claudin-1 were detected at the tight junctions of villous enterocytes in each group ( $\mathrm{n}=10$ ). Scale bar= $50 \mu \mathrm{m}$. The Western blotting assay revealed the expression levels of (B) ZO-1 and (C) claudin- 1 and a housekeeping protein $(\beta$-actin) in the duodenum tissue from each group of rats $(n=6)$. ${ }^{*} p<0.05, \mathrm{HS}+\mathrm{R}+\mathrm{RJVF} 36{ }^{\circ} \mathrm{C}$ vs. non-HS+non-R+RJVF $36^{\circ} \mathrm{C} ;+p<0.05, \mathrm{HS}+\mathrm{R}+\mathrm{RJVF} 4{ }^{\circ} \mathrm{C}$ vs. HS+R+RJVF $36^{\circ} \mathrm{C}$. 
(A)
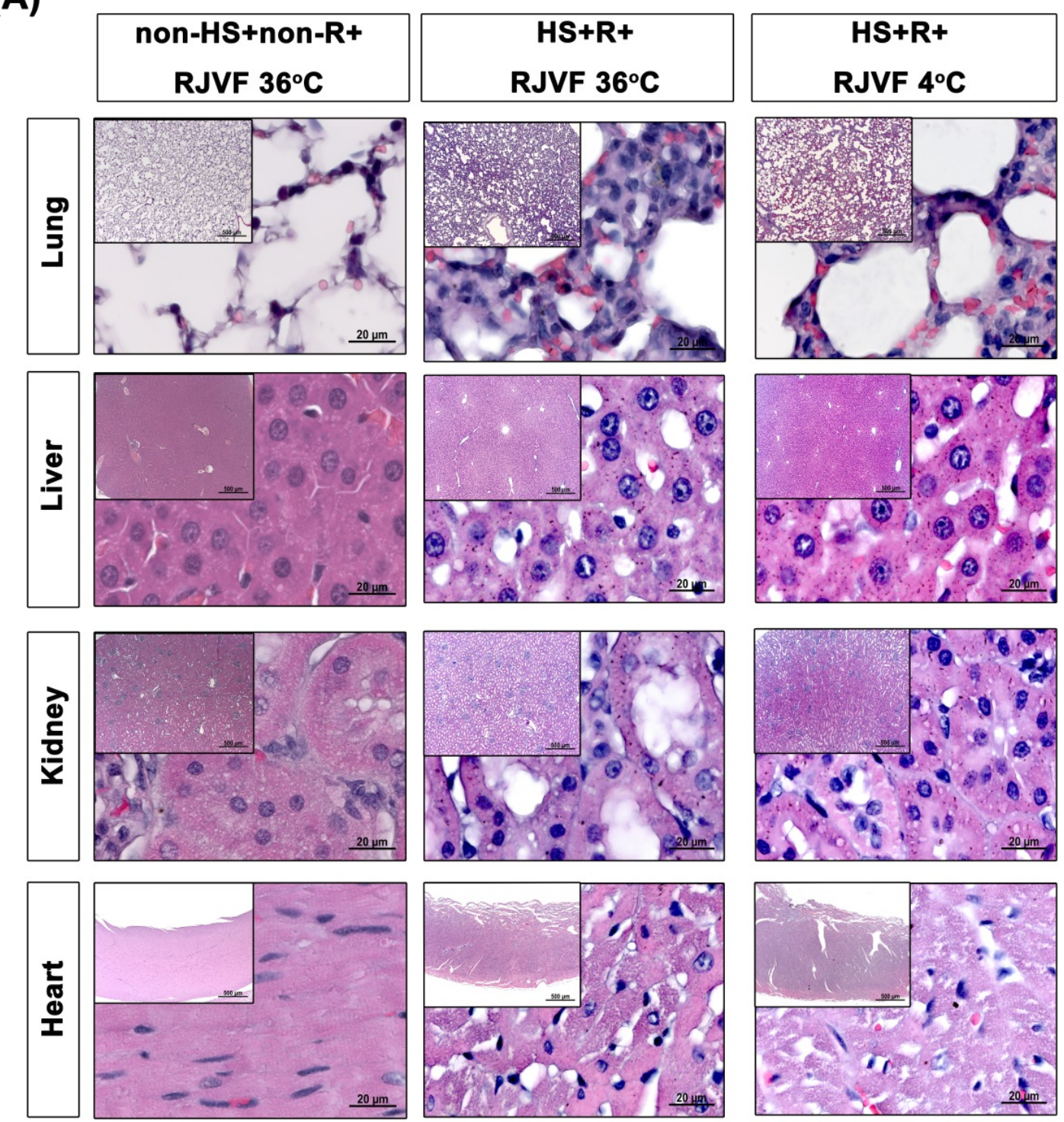

(B)

\section{H-E stain (Multiple organs)}
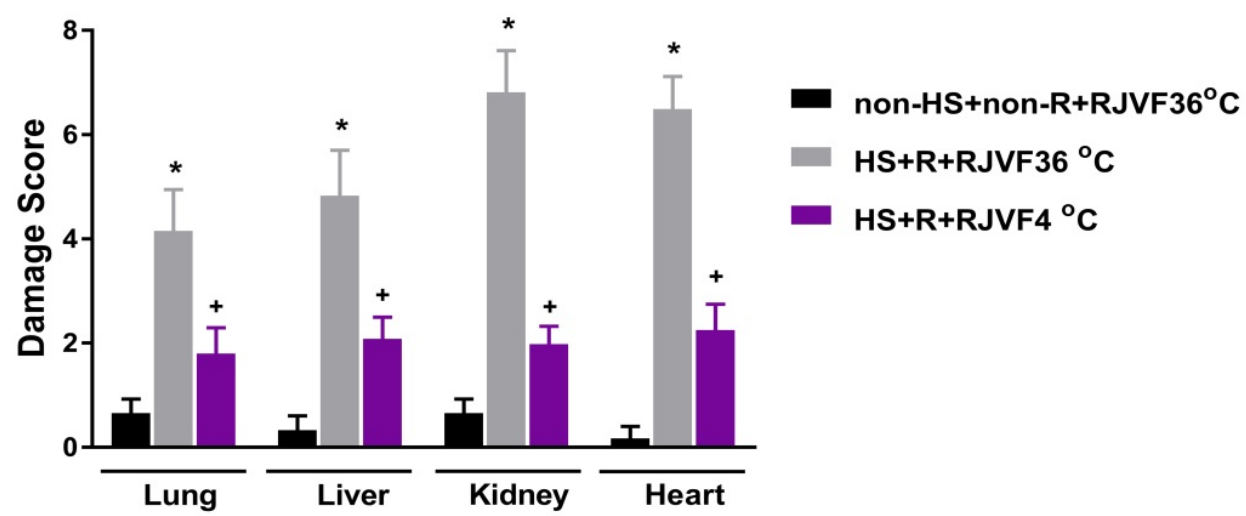

Figure 4. Multiple organ damage after hemorrhagic resuscitation. (A) Representative photographs of $\mathrm{H} \& \mathrm{E}$ staining revealed that $\mathrm{HS}$ rats showed cell infiltration, pulmonary edema, and alveolar collapse in the lung tissue, disorganized hepatocyte, sinusoidal dilation and necrotic areas in the liver tissues, and altered glomeruli structure (atrophic and dysmorphic), altered tubular structure, altered tubular epithelial cells, vascular dilation/ congestion, increased number of inflammatory cells, intracytoplasmic vacuoles, hemorrhage and inflammatory cell infiltration in the cardiomyocytes. (B) The tissue damage scores for different organs. Each bar represents the mean \pm S.D. of 10 rats. ${ }^{*} p<0.05$, the HS+R+RJVF $36{ }^{\circ} \mathrm{C}$ group vs. the non-HS+non-R+RJVF $36{ }^{\circ} \mathrm{C}$ group; $+p<0.05$, the HS+R+RJVF $4{ }^{\circ} \mathrm{C}$ group vs. the HS+R+RJVF $36{ }^{\circ} \mathrm{C}$ group. Scale bar $=500 \mu m$ and $20 \mu \mathrm{m}$. The images of multiple organs morphology at $50 \times$ magnification and $1000 \times$ magnification were shown, respectively. 


\section{Discussion}

Our present result showed that an HS caused systemic inflammation, gut barrier disruption, and peripheral organ damage. Additionally, we found that EJ-based infusion cooled the brain robustly with a minimal effect on body temperatures. This brain cooling significantly reduced systemic inflammation, gut barrier, and peripheral organ damage in HS rats.

Cannulation of the internal jugular vein is a frequently conducted bedside procedure for placing central venous catheters [18]. A more recent report showed that internal jugular infusion of cold saline conferred hypothermic and neuroprotective effects in ischemic stroke [10]. Compared to the human cerebral venous system, the rat's internal jugular vein is relatively thin for cannulation experiments, and the EJ is the main vessel for cerebral venous drainage, which has a relatively greater diameter for cannulation [11]. In this study, we used the external jugular vein for cannulation and found that the cooling and neuroprotective efficacy of the EJ infusion of cold saline was comparable to whole-body cooling by many metrics evaluated [2]. Because jugular vein puncture is an easy procedure frequently used in the emergency department, we speculated that hypothermic jugular flush would be an alternative to achieve rapid brain cooling, especially in traumatized patients with an HS [7]. However, the EJ is the predominant drainage of the face and head, not the brain. It is bigger than the internal jugular (IJ) vein in non-human mammals because their brains are smaller relative to the face. There are striking differences, including the direction (normal IJ cannulation is antegrade vs. retrograde for this cooling method, and having to accurately direct a catheter towards the cerebrum to deliver cold crystalloid seems much more difficult than placing a standard central venous catheter).

Whole-body cooling $\left(32-34{ }^{\circ} \mathrm{C}\right)$ or therapeutic hypothermia improves the outcome of HS by reducing the resuscitation fluid volume required to maintain blood pressure [19], the expression of reactive oxygen species as well as microvascular permeability [20]. Whole-body cooling may exert its beneficial effects by affecting nearly every metabolic, molecular, and cellular event in cell death, ultimately promoting tissue preservation and repair [21]. However, the adverse reactions following a whole-body cooling may limit its use in treating HS injury. Probably, the most striking findings of the present study are that EJ infusion of cold saline induces cooling to only the brain $\left(32-33^{\circ} \mathrm{C}\right)$, without the body core $\left(\sim 37^{\circ} \mathrm{C}\right)$, thus circumventing any adverse systemic-effects which may result from total body cooling. Indeed, our present study demonstrates that selective brain cooling induces cooling to the brain, which reduces intestine and other peripheral organ injuries in rats with HS resuscitation. By using the intestine damage scores, we compare the beneficial effects of therapeutic hypothermia between the systemic hypothermia and selective brain cooling. Mucosal damage was classified as follows: 0 (normal), 1 (surface epithelium damaged), 2 ( $<50 \%$ mucosa damaged), 3 (>50\% mucosa damaged), and 4 (entire mucosa damaged). Our previous results showed that whole body cooling $\left(32-34^{\circ} \mathrm{C}\right)$ reduced the intestinal damage scores from 3-4 to 2-3 in the rats with HS. In our present results, brain cooling $\left(32-34{ }^{\circ} \mathrm{C}\right)$ shared with whole body cooling the similar efficiency (decreased the intestine damage score from 3-4 to 2-3). The RJVF $4{ }^{\circ} \mathrm{C}$ saline infusion can lead to protection with a shorter cooling duration and lower the temperatures by only $3-4{ }^{\circ} \mathrm{C}$. Thus, selective brain cooling would be an attractive alternation to whole body cooling [22, 23]. We speculate there is a direct effect to protect organ damage directly from brain specific hypothermia or it is a byproduct of preserving intestinal epithelial hyperpermeability.

Our present study showed that rats with resuscitation from HS exhibited both defective tight junctions (evidenced by altered expression of the transmembrane proteins ZO-1 and claudin-1) and intestinal epithelium hyperpermeability (or increased FD-4 clearance). Activated transcellular mechanisms increase intestinal permeability to macromolecules such as nutrients, proteins, and bacteria [24]. In line with our findings, it has been shown that resuscitation from HS or traumatic brain injury causes disruption of the intestinal epithelial barrier accompanied by decreased expression of tight junction proteins ZO-1 and occludin- 1 in the intestine $[25,26]$. In our present results, selective brain cooling attenuates the loss of intestinal epithelial layer integrity, the increased release of pro-inflammatory cytokines, and multiple organ injuries, perhaps via the brain-gut axis [27, 28].

In fact, the "three-hit model" theory proposed by previous studies $[9,27,29]$ is partially confirmed by our present results. An initial HS insult causes splanchnic hypoperfusion; the intestine responds by producing and releasing pro-inflammatory cytokines. Blood transfusion resuscitation causes reperfusion and results in both ischemia/reperfusion injury to the intestine and intestinal epithelial hyperpermeability and augments intestinal inflammatory responses. Translocation of bacterias and endotoxins from intestines to the bloodstream further enhances the immune responses with the release of inflammatory cytokines and other mediators both locally and systemically and results in multiple organ deficiency 
syndromes [27]. The central nervous system (CNS) and peripheral tissues are connected by the peripheral nervous system (PNS), which transmits various signals via neurotransmitters to affect immune reactions.

\section{Conclusions}

Following HS, rats caused visceral hypoperfusion, intestinal ischemia/reperfusion injury, gut barrier disruption, systemic inflammation, and peripheral vital organ injuries. Intrajugular-based infusion cooled the brain robustly with a minimal effect on body core temperatures. This brain cooling led to significantly reduced gut barrier disruption, systemic inflammation, and peripheral vital organ injuries in rats. Compared with whole-body cooling, EJ infusion conferred a similar degree of peripheral vital organ protection following HS in rats requiring blood transfusion via preserving the integrity of the brain-gut axis.

\section{Abbreviations}

ALP: alkaline phosphatase; ALT: alanine aminotransferase; AST: aspartate aminotransferase; BBB: blood-brain barrier; BUN: blood urea nitrogen; CBF: cerebral blood flow; DAPI: 4,5-diamidino-2phenylindole; EJ: external jugular; ELISA: enzymelinked immunosorbent assay; FD-4: fluoresceinisothiocyanate dextran; HR: heart rate; HS: hemorrhagic shock; IACUC: institutional animal care and use committee; IJ: internal jugular; IL-1 $\beta$ : interleukin-1 beta; IL-18: interleukin-18; IL-6: interleukin-6; MABP: mean arterial blood pressure; PVDF: polyvinylidene fluoride; RJVF: retrograde jugular vein flush; SBF: striatal blood flow; SDS-PAGE: sodium dodecyl sulfate-polyacrylamide; $\mathrm{Tb}$ : brain temperature; Tco: colon temperature; TNF- $\alpha$ : tumor necrosis factor-alpha; WHC: whole-body cooling.

\section{Supplementary Material}

Supplementary table S1.

http://www.medsci.org/v18p2920s1.pdf

\section{Acknowledgments}

We acknowledge Ms. Meng-Tsung Ho for her excellent editorial assistance in manuscript preparation.

\section{Funding}

This study was supported by the Ministry of Science and Technology (Taiwan) (grant no. MOST109-2314-B-384-002-MY3; MOST108-2314-B384-006-MY2; and MOST 104-2314-B-384-003-MY3), Chi Mei Medical Center (Taiwan) (grant no.
CMFHT10802; CMORA10904), and Chi Mei Medical Center, Liouying (Taiwan) (grant no. CLFHR10732; CLFHR10825). The funders had no role in the study design, data collection and analysis, the decision to publish, or preparation of the manuscript. The contents of the manuscript are solely the responsibility of the authors and do not necessarily represent the official views of the Taiwan MOST and Chi Mei Medical Center.

\section{Data and materials availability}

The authors confirm that the data supporting the findings of this study are available within the article.

\section{Author contribution}

Study concept and design (CMC, $\mathrm{CCH}, \mathrm{CCC})$; acquisition of data (CCHu, $\mathrm{CHW}, \mathrm{CPC})$; analysis and interpretation of data (HJL, CHW, MTL); drafting of the manuscript $(\mathrm{CPC}, \mathrm{HJL}, \mathrm{CCC})$; critical revision of the manuscript for important intellectual content (CMC, HJL, MTL); statistical analysis (CHW, CCH); material support (CCC, CPC); obtained funding (CMC, CCC, CPC).

\section{Competing Interests}

The authors have declared that no competing interest exists.

\section{References}

1. Cannon JW. Hemorrhagic Shock. New England Journal of Medicine. 2018; 378: 370-9.

2. Chio CC, Hsu CC, Tian YF, Wang CH, Lin MT, Chang CP, et al. Combined hemorrhagic shock and unilateral common carotid occlusion induces neurological injury in adult male rats. International journal of medical sciences. 2017; 14: 1327-34.

3. Wrba L, Ohmann JJ, Eisele P, Chakraborty S, Braumuller S, Braun CK, et al. Remote intestinal injury early after experimental polytrauma and hemorrhagic shock. Shock (Augusta, Ga). 2018.

4. Zhou J, Huang WQ, Li C, Wu GY, Li YS, Wen SH, et al. Intestinal ischemia/reperfusion enhances microglial activation and induces cerebral injury and memory dysfunction in rats. Critical care medicine. 2012; 40: 2438-48.

5. Natanov R, Gueler F, Falk CS, Kuhn C, Maus U, Boyle EC, et al. Blood cytokine expression correlates with early multi-organ damage in a mouse model of moderate hypothermia with circulatory arrest using cardiopulmonary bypass. PloS one. 2018; 13: e0205437.

6. Polderman KH, Herold I. Therapeutic hypothermia and controlled normothermia in the intensive care unit: practical considerations, side effects, and cooling methods. Critical care medicine. 2009; 37: 1101-20.

7. Ferrara A, MacArthur JD, Wright HK, Modlin IM, McMillen MA. Hypothermia and acidosis worsen coagulopathy in the patient requiring massive transfusion. American journal of surgery, 1990; 160: 515-8.

8. Brzozowski B, Mazur-Bialy A, Pajdo R, Kwiecien S, Bilski J, Zwolinska-Wcislo $\mathrm{M}$, et al. Mechanisms by which Stress Affects the Experimental and Clinical Inflammatory Bowel Disease (IBD): Role of Brain-Gut Axis. Current neuropharmacology. 2016; 14: 892-900.

9. Chang JX, Chen S, Ma LP, Jiang LY, Chen JW, Chang RM, et al. Functional and morphological changes of the gut barrier during the restitution process after hemorrhagic shock. World journal of gastroenterology. 2005; 11: 5485-91.

10. Duan Y, Wu D, Huber M, Shi J, An H, Wei W, et al. New Endovascular Approach for Hypothermia With Intrajugular Cooling and Neuroprotective Effect in Ischemic Stroke. Stroke. 2020; 51: 628-36.

11. Wen YS, Huang MS, Lin MT, Lee CH. Hypothermic retrograde jugular vein flush in heatstroke rats provides brain protection by maintaining cerebral blood flow but not by hemodilution. Critical care medicine. 2004; 32: 1391-5.

12. Dietrich WD, Bramlett HM. Therapeutic hypothermia and targeted temperature management in traumatic brain injury: Clinical challenges for successful translation. Brain research. 2016; 1640: 94-103. 
13. Kuo JR, Lo CJ, Chang CP, Lin HJ, Lin MT, Chio CC. Brain cooling-stimulated angiogenesis and neurogenesis attenuated traumatic brain injury in rats. The Journal of trauma. 2010; 69: 1467-72.

14. McBride DW, Wu G, Nowrangi D, Flores JJ, Hui L, Krafft PR, et al. Delayed Recanalization Promotes Functional Recovery in Rats Following Permanent Middle Cerebral Artery Occlusion. Transl Stroke Res. 2018; 9: 185-98.

15. Zhao B, Fei J, Chen Y, Ying YL, Ma L, Song XQ, et al. Pharmacological preconditioning with vitamin $\mathrm{C}$ attenuates intestinal injury via the induction of heme oxygenase-1 after hemorrhagic shock in rats. PloS one. 2014; 9: e99134.

16. Tan SJ, Yu C, Yu Z, Lin ZL, Wu GH, Yu WK, et al. High-fat enteral nutrition reduces intestinal mucosal barrier damage after peritoneal air exposure. The Journal of surgical research. 2016; 202: 77-86.

17. Fredenburgh LE, Velandia MM, Ma J, Olszak T, Cernadas M, Englert JA, et al. Cyclooxygenase-2 deficiency leads to intestinal barrier dysfunction and increased mortality during polymicrobial sepsis. Journal of immunology (Baltimore, Md : 1950). 2011; 187: 5255-67.

18. Felleiter P, Gustorff B, Lierz P, Hörauf K. [Use of electrocardiographic placement control of central venous catheters in Austria]. Acta medica Austriaca. 1999; 26: 109-13.

19. Nishi K, Takasu A, Shibata M, Uchino S, Yamamoto $Y$, Sakamoto T. Hypothermia reduces resuscitation fluid volumes required to maintain blood pressure in a rat hemorrhagic shock model. The journal of trauma and acute care surgery. 2012; 72: 130-5.

20. Childs EW, Udobi KF, Hunter FA. Hypothermia reduces microvascular permeability and reactive oxygen species expression after hemorrhagic shock. The Journal of trauma. 2005; 58: 271-7

21. Kurisu K, Yenari MA. Therapeutic hypothermia for ischemic stroke; pathophysiology and future promise. Neuropharmacology. 2018; 134: 302-9.

22. Busto R, Dietrich WD, Globus MY, Valdes I, Scheinberg P, Ginsberg MD. Small differences in intraischemic brain temperature critically determine the extent of ischemic neuronal injury. J Cereb Blood Flow Metab. 1987; 7: 729-38.

23. Esposito E, Ebner M, Ziemann U, Poli S. In cold blood: intraarteral cold infusions for selective brain cooling in stroke. J Cereb Blood Flow Metab. 2014; 34: 743-52.

24. Barreau $\mathrm{P}$, Huger J. Intestinal barrier dysfunction triggered by invasive bacteria. Curr Opin Microbiol. 2014; 17: 8.

25. Bansal V, Costantini T, Kroll L, Peterson C, Loomis W, Eliceiri B, et al. Traumatic brain injury and intestinal dysfunction: uncovering the neuro-enteric axis. Journal of neurotrauma. 2009; 26: 1353-9.

26. Bansal V, Ryu SY, Blow C, Costantini T, Loomis W, Eliceiri B, et al. The hormone ghrelin prevents traumatic brain injury induced intestinal dysfunction. Journal of neurotrauma. 2010; 27: 2255-60.

27. Sertaridou E, Papaioannou V, Kolios G, Pneumatikos I. Gut failure in critical care: old school versus new school. Annals of gastroenterology. 2015; 28: 309-22.

28. Katzenberger RJ, Ganetzky B, Wassarman DA. The gut reaction to traumatic brain injury. Fly. 2015; 9: 68-74.

29. Deitch EA. Bacterial translocation or lymphatic drainage of toxic products from the gut: what is important in human beings? Surgery. 2002; 131: 241-4 